\title{
Maternal nicotine dependence is associated with longitudinal increases in child obesogenic eating behaviors
}

\author{
Jenna R. Cummings ${ }^{1,2}$ (D) | Ashley N. Gearhardt ${ }^{1,2}$ | Alison L. Miller ${ }^{2,3}$ | Luke W. Hyde ${ }^{1,2}$ | \\ Julie C. Lumeng ${ }^{2,4}$
}

${ }^{1}$ Department of Psychology, University of Michigan, Ann Arbor, Michigan, USA

${ }^{2}$ Center for Human Growth and Development, University of Michigan, Ann Arbor, Michigan, USA

${ }^{3}$ School of Public Health, University of Michigan, Ann Arbor, Michigan, USA

${ }^{4}$ Department of Pediatrics, University of Michigan, Ann Arbor, Michigan, USA

\section{Correspondence}

Jenna R. Cummings, Department of Psychology, University of Michigan, 1465 East Hall, 530 Church St., Ann Arbor, MI 48109, USA.

E-mail: jennarc@umich.edu

Funding information

University of Michigan Ann Arbor, Grant/ Award Number: MCubed Grant; National Institute of Diabetes and Digestive and Kidney Diseases, Grant/Award Numbers: R01DK098983, R21DK090718 and RC1DK086376; Eunice Kennedy Shriver National Institute of Child Health and Human Development, Grant/Award Numbers: R01HD061356 and T32HD079350

\author{
Summary \\ Background: Meta-analysis shows that parental cigarette smoking is associated \\ with child obesity. \\ Objectives: This study tested for associations between severity of maternal nico- \\ tine dependence and longitudinal changes in child eating behavior in archival data \\ analysis.
}

Methods: Maternal nicotine dependence was assessed with the Fagerstrom Test for Nicotine Dependence. Child eating behavior was assessed with the Child Eating Behavior Questionnaire when children were ages 4, 6, 8, and 10.

Results: Over and above the influence of child age, child biological sex, and family income-to-needs ratio, more severe maternal nicotine dependence was associated with greater increases in child Food Responsiveness $\left(\gamma=0.07, S E_{\gamma}=0.03, P=.014\right.$, $95 \% \mathrm{Cl}[0.01,0.13])$ and Emotional Overeating $\left(\gamma=0.06, S E_{\gamma}=0.03, P=.024,95 \%\right.$ $\mathrm{Cl}[0.01,0.11])$ across 6 years.

Conclusions: Maternal nicotine dependence may be a transdiagnostic risk factor that identifies children at risk for reward-driven, obesogenic eating behavior.

\section{KEYWORDS}

Childhood, eating behavior, longitudinal, parental nicotine dependence

\section{1 | INTRODUCTION}

Over 20 million US adults smoke tobacco cigarettes. ${ }^{1}$ The majority of these smokers do not quit, ${ }^{2}$ and individuals do not "mature out" of cigarette smoking during adulthood the way they do with use of other addictive substances. ${ }^{3}$ Adults who smoke thus represent a group highly vulnerable to addictive substances.

Critically, children of adults who smoke are likewise vulnerable to addictive substances. Parental cigarette smoking is associated with greater likelihood of adolescent (ages 12-17) initiation of cigarette smoking, ${ }^{4}$ and with greater adolescent use of other substances including alcohol and marijuana. ${ }^{5}$ Maternal (versus paternal) substance use particularly shows robust associations with adolescent substance use. $^{4,5}$ Thus, children of parents (especially mothers) who smoke are generally vulnerable to substance use.

Evidence is building that rewarding aspects of addictive substances overlap with those of processed foods high in refined carbohydrates and fat ${ }^{6,7}$-foods that are now widely available in the "toxic" food environment and that promote obesogenic eating (ie, eating in a way that is a risk factor for obesity). ${ }^{8}$ Thus, children of parents (especially mothers) who smoke may also be vulnerable to obesogenic eating.

Indeed, a meta-analysis of 12 studies indicates that parental cigarette smoking is associated with youth (ages 3-18) obesity, with maternal (versus paternal) cigarette smoking demonstrating stronger influence. ${ }^{9}$ However, there is a gap in understanding how parental cigarette smoking associates with child eating behavior. This gap is 
important to fill to guide prevention efforts. Child eating behavior is a potentially modifiable target for improving lifelong physical health. ${ }^{10}$ Moreover, there are several types of eating behaviors observed in children (eg, Food Responsiveness, Emotional Overeating, Satiety Responsiveness), with some especially obesogenic (eg, Food Responsiveness, Emotional Overeating). ${ }^{11}$ Understanding which eating behaviors are associated with parental cigarette smoking may shed light on potential pathways tying this risk factor to child obesity.

The present study adds to the literature by investigating whether severity of maternal nicotine dependence is associated with longitudinal changes in child eating behavior. Maternal nicotine dependence was investigated given that maternal versus paternal cigarette smoking shows stronger associations with child obesity. ${ }^{9}$ Associations with longitudinal changes in child eating behavior were tested because as children age they are exposed to new food environments (eg school, friends' homes) that potentially provide greater access to processed foods high in refined carbohydrates and fat. Child obesogenic eating is therefore likely to emerge over time. Our hypothesis was that more severe maternal nicotine dependence would be associated with greater longitudinal increases in child obesogenic eating.

\section{2 | METHODS}

This study was an archival data analysis of data from children $(N=57)$ who participated in four waves of the Appetite, Behavior, and Cortisol study at ages 4, 6, 8, and $10 .{ }^{12}$ Children were recruited from Head Start, a federally funded preschool program for families whose income falls below federal poverty guidelines; full recruitment and exclusion details are reported elsewhere. ${ }^{12}$ The University of Michigan
Institutional Review Board approved procedures in accordance with the World Medical Association Declaration of Helsinki. Caretakers provided written informed consent while children provided ageappropriate verbal assent.

At Wave 4, caretakers reported on nicotine dependence. The present study's final sample was restricted to children who had a mother who reported on nicotine dependence $(n=50)$. Nicotine dependence was indexed by a summed continuous score (0-10) from yes/no (0-1) and multiple-choice items (0-3) on the Fagerstrom Test for Nicotine Dependence, ${ }^{13}$ which measures current symptoms of substance use disorder specific to cigarette smoking (ie, quantity of cigarettes smoked, compulsion to use). Mothers who reported that they did not currently smoke cigarettes (59.2\%) received a score of 0 , and higher total scores indicated more severe maternal nicotine dependence. Descriptives are presented in Table 1; 10.2\%, 14.3\%, 10.2\%, and $2.0 \%$ mothers demonstrated low, low to moderate, moderate, and high dependence symptoms, respectively.

At Waves 1-4, caretakers completed the Child Eating Behavior Questionnaire, ${ }^{10}$ which is a 35 -item informant report of eating behaviors observed in children. Responses to items ranged from 1 (Never) to 5 (Always). Multilevel growth modeling was conducted to confirm that average values of each child eating behavior significantly changed over time, and multilevel estimates are provided in Table 1. Food Responsiveness (appetitive drive and food cue responsiveness; $\alpha_{\mathrm{t1}-\mathrm{t} 4}=.78$, $.78, .83, .81$ ) and Emotional Overeating (eating more in response to negative affect; $\alpha_{\mathrm{t} 1-\mathrm{t} 4}=.77, .78, .85, .83$ ) increased over time. Emotional Undereating (eating less in response to negative affect; $\alpha_{\mathrm{t} 1-\mathrm{t} 4}=.70, .62, .70, .71$ ), Satiety Responsiveness (sensitivity to internal satiety signals; $\alpha_{\mathrm{t} 1-\mathrm{t} 4}=.73, .73, .69, .73$ ), and Desire to Drink (drive for non-alcoholic beverages; $\alpha_{\mathrm{t1}-\mathrm{t} 4}=.87, .90, .88, .92$ ) decreased

TABLE 1 Sample demographics, variable descriptives, and multilevel estimates of time slopes for child eating behavior $(n=50)$

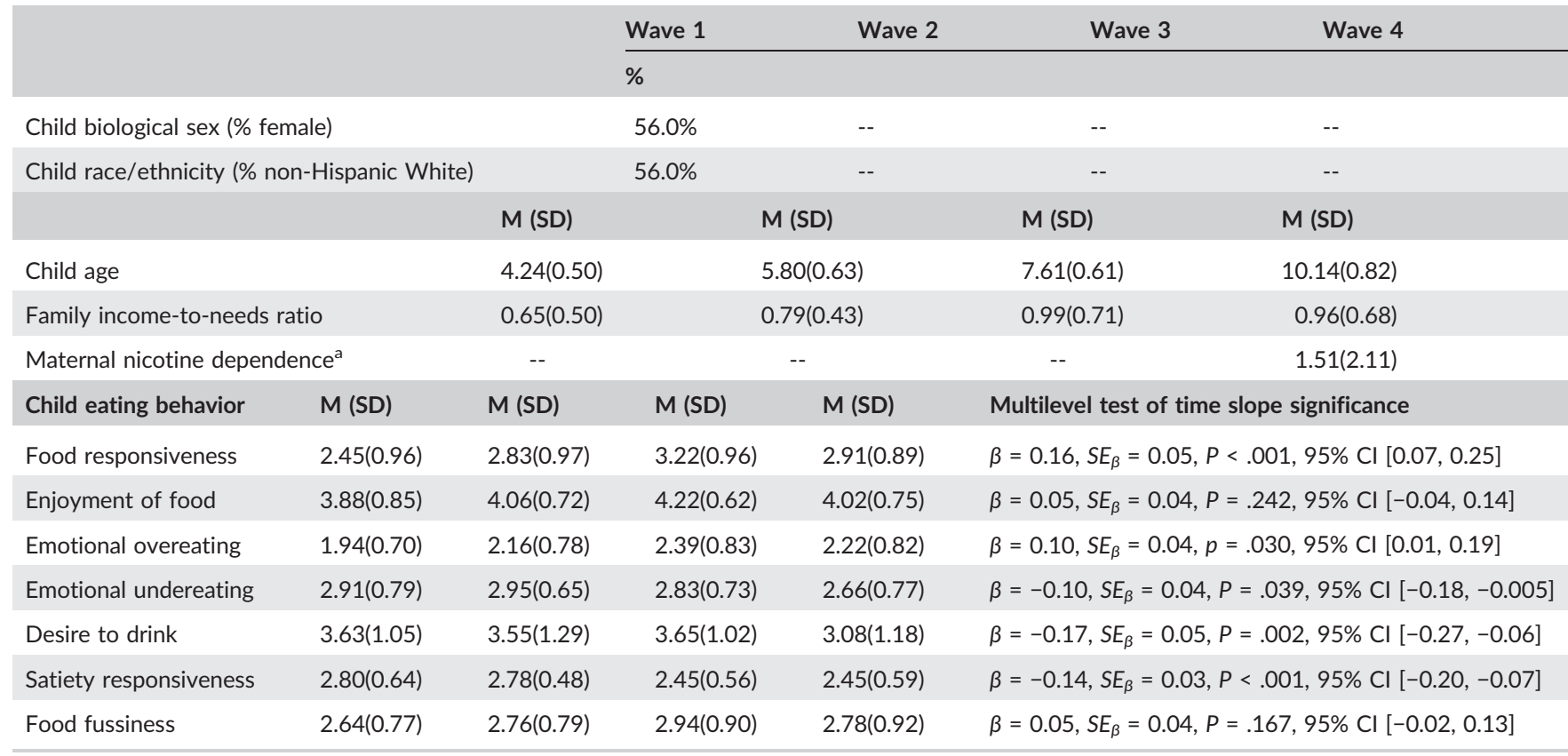

${ }^{a}$ Maternal nicotine dependence was indexed by a summed continuous score (0-10) on the Fagerstrom Test for Nicotine Dependence. ${ }^{13} 59.2 \%, 10.2 \%$, $14.3 \%, 10.2 \%$, and $2.0 \%$ mothers demonstrated no, low, low to moderate, moderate, and high dependence symptoms, respectively. 
over time. Enjoyment of Food (interest in food; $\alpha_{\mathrm{t} 1-\mathrm{t} 4}=.83, .80, .80$, .87) and Food Fussiness (selectivity about which foods are eaten; $\alpha_{\mathrm{t} 1 \mathrm{t} 4}=.85, .85, .88, .87$ ) did not change over time. Given our hypothesis was to examine associations between maternal nicotine dependence and longitudinal changes in eating behavior, analyses were restricted to eating behaviors that changed over time.

To test how maternal nicotine dependence was associated with child eating behavior over time, multilevel growth modeling was conducted with time entered at Level 1. Each child eating behavior was a separate dependent variable. Time was centered such that the model's slope represented changes in child eating behavior from age 4 to 10 . Child age and family income-to-needs ratio were entered as time varying covariates at Level ${ }^{11}$; child biological sex was entered as a time invariant covariate entered at Level $2 .{ }^{10}$ Random effects for time intercept and slope were included in all models except Food Responsiveness and Desire to Drink because those models fit better as indicated by a significantly smaller $-2 L L$. The Food Responsiveness and Desire to Drink models fit better with random effects for time intercept only. All model estimations were conducted in SAS University Edition using the restricted maximum likelihood estimation approach, which accounts for missing data ( $<6 \%$ data were missing).

\section{3 | RESULTS}

Sample demographics are presented in Table 1. Multilevel estimates from hypothesis testing are presented in Table 2. More severe maternal nicotine dependence was significantly associated with greater increases in child Food Responsiveness and Emotional Overeating over 6 years. There were no significant associations of maternal nicotine dependence with decreases in child Emotional Undereating, Satiety Responsiveness, and Desire to Drink over 6 years.

\section{4 | DISCUSSION}

Cigarette smoking remains a prevalent and persistent behavior among US adults, ${ }^{1-3}$ and children of parents (particularly mothers) who smoke are generally vulnerable to addictive substances. ${ }^{4,5}$ Given that rewarding aspects of substances may overlap with those of processed foods high in refined carbohydrates and fat, ${ }^{6,7}$ the present study used archival data analysis to test the hypothesis that maternal nicotine dependence would be associated with greater longitudinal increases in obesogenic eating behaviors. Over and above the influence of child age, child biological sex, and family incometo-needs ratio, maternal nicotine dependence was associated with greater increases in child Food Responsiveness and child Emotional Overeating across 6 years. Importantly, child Food Responsiveness and child Emotional Overeating are strongly associated with greater child body mass index in prior work. ${ }^{11}$

Just as children of adults who smoke may inherit vulnerability toward substance use, ${ }^{4,5}$ the present study results suggest that they may inherit vulnerability toward obesogenic eating because of overlap in rewarding aspects of substances and certain foods. ${ }^{6,7}$ Indeed, maternal nicotine dependence was selectively associated with increases in child eating behaviors that are reward driven (ie, Food Responsiveness, Emotional Overeating). ${ }^{14}$ However, multiple additional mechanisms may explain these results. First, families including mothers who smoke may experience greater family stress, which may contribute to child reward-driven, obesogenic eating. ${ }^{15}$ Second, mothers who smoke may exhibit risk factors for obesity themselves including altered chemosensation, increased fat/carbohydrate liking, poor diet, dietary restraint, and altered food reinforcement ${ }^{16,17}$; thus, they may provide a poor diet to children and/or model rewarddriven, obesogenic eating. Third, mothers who smoke may expose children prenatally and during lactation, which-as shown in rodent models-may alter motivational drives for high-fat foods (although our measure of nicotine dependence did not ask about smoking

TABLE 2 Multilevel estimates from models testing associations between parental nicotine dependence and longitudinal changes in child eating behavior $(n=50)$

$\gamma \quad \mathrm{SE}_{\gamma}$

$95 \% \mathrm{C}$

Lower Upper

Child food responsiveness

Time $\times$ maternal nicotine dependence ${ }^{a}$ 0.07 0.03 .014 0.01 0.13

Child emotional overeating

Time $\times$ maternal nicotine dependence ${ }^{a}$

.024

0.01

0.11

Child emotional undereating

Time $\times$ maternal nicotine Dependence ${ }^{a}$

$-0.03$

0.03

.339

$-0.09$

Child satiety responsiveness

Time $\times$ maternal nicotine dependence ${ }^{a}$

$-0.02$

0.02

251

$-0.06$

0.02

Child desire to drink

Time $\times$ maternal nicotine dependence ${ }^{\mathrm{a}}$ 0.02

0.03

.569

$-0.05$

0.08

Child biological sex was entered as a time invariant covariate entered at Level 2. Results are statistically significant where $P<.05$. 
during these periods). ${ }^{18}$ Critically, biologically inherited vulnerabilities, family stress, poor diet selection, parental modeling, and prenatal exposures may collectively place children of mothers who smoke at risk for obesogenic eating.

There are limitations that should be considered when interpreting results. Our index of maternal nicotine dependence is well validated ${ }^{13}$ but biomarkers exist (eg, plasma/hair cotinine). ${ }^{19}$ This index also was not collected at baseline, so although most smokers do not quit or "mature out" of cigarette smoking, ${ }^{2,3}$ it is unclear what symptoms mothers demonstrated at baseline. The sample size was relatively small; however, multilevel models with sample sizes $\geq 50$ at the highest level of analysis reduce likelihood of biased estimates. ${ }^{20}$

In sum, maternal nicotine dependence may be a transdiagnostic risk factor that identifies children at risk for reward-driven, obesogenic eating behavior. Future research should identify the precise mechanisms by which this occurs.

\section{ACKNOWLEDGEMENTS}

J.R.C. conducted the literature search, analyzed data, interpreted data, generated tables, and wrote the initial draft of the manuscript. A.N.G. oversaw data analysis and interpretation. A.N.G., A.L.M., L.W.H., and J. C.L. oversaw data collection. All authors were involved in writing the paper and provided final approval of the manuscript.

J.R.C. was supported by Award Number T32HD079350 from the Eunice Kennedy Shriver National Institute of Child Health and Human Development. The present research was supported by Award Number R01HD061356 from the Eunice Kennedy Shriver National Institute of Child Health and Human Development, and by Award Numbers R21DK090718, R01DK098983, and RC1DK086376 from the National Institute of Diabetes and Digestive and Kidney Diseases. The present research was also supported by a MCubed grant from the University of Michigan to J.C.L., L.W.H., and A.N.G. The content of this manuscript is solely the responsibility of the authors and does not necessarily represent the official views of the Eunice Kennedy Shriver National Institute of Child Health and Human Development, the National Institute of Diabetes and Digestive and Kidney Diseases, or the National Institutes of Health.

\section{CONFLICT OF INTEREST}

No conflict of interest was declared.

\section{ORCID}

Jenna R. Cummings (D) https://orcid.org/0000-0001-8124-2666

\section{REFERENCES}

1. Jamal A, Phillips E, Gentzke AS, et al. Current cigarette smoking among adults-United States, 2016. Morb Mortal Wkly Rep 2018, 67: 53-59.

2. Chassin L, Presson CC, Pitts SC, Sherman SJ. The natural history of cigarette smoking from adolescence to adulthood in a Midwestern community sample: multiple trajectories and their psychosocial correlates. Health Psychol 2000; 19: 223-231 https://doi.org/ 10.1037/TO7S-6133.19.3.223[published Online First: Epub Date].

3. Chen K, Kandel DB. The natural history of drug use from adolescence to the mid-thirties in a general population sample. Am J Public Health 1995; 85: 41-47.

4. Gilman SE, Rende R, Boergers J, et al. Parental smoking and adolescent smoking initiation: an intergenerational perspective on tobacco control. Pediatrics 2009; 123: 274-281 https://doi.org/10.1542/peds.20082251[published Online First: Epub Date].

5. Andrews JA, Hops H, Ary D, Tildesley E, Harris J. Parental influence on early adolescent substance use: specific and nonspecific effects. J Early Adolesc 1993; 13: 285-310.

6. Schulte EM, Avena NM, Gearhardt AN. Which foods may be addictive? The roles of processing, fat content, and glycemic load. PLOS ONE 2015; 10: e0117959 https://doi.org/10.1371/journal.pone.0117959 [published Online First: Epub Date.

7. Falbe J, Thompson HR, Patel A, Madsen KA. Potentially addictive properties of sugar-sweetened beverages among adolescents. Appetite 2018; 133: 130-137 https://doi.org/10.1016/j.appet.2018.10.032 [published Online First: Epub Date].

8. Brownell KD. The environment and obesity. In: Fairburn CG, Brownel KD (eds). Eating Disorders and Obesity: A Comprehensive Handbook. Second Edition ed. Guilford Publications: New York, NY, 2002, pp. 433-438.

9. Riedel C, Schönberger K, Yang S, et al. Parental smoking and childhood obesity: higher effect estimates for maternal smoking in pregnancy compared with paternal smoking-a meta-analysis. Int J Epidemiol 2014; 43: 1593-1606 https://doi.org/10.1093/ije/dyu150.

10. Wardle J, Guthrie CA, Sanderson S, Rapoport L. Development of the Children's Eating Behaviour Questionnaire. J Child Psychol Psychiatry Allied Discip 2001; 42: 963-970 https://doi.org/10.1017/ S0021963001007727[published Online First: Epub Date].

11. Webber L, Hill C, Saxton J, Van Jaarsveld CH, Wardle J. Eating behaviour and weight in children. Int J Obes (Lond) 2009; 33: 21-28 https://doi.org/10.1038/ijo.2008.219[published Online First: Epub Date].

12. Lumeng JC, Miller A, Peterson KE, et al. Diurnal cortisol pattern, eating behaviors and overweight in low-income preschool-aged children. Appetite 2014; 73: 65-72 https://doi.org/10.1016/j.appet.2013.10. 016[published Online First: Epub Date.

13. Heatherton TF, Kozlowski LT, Frecker RC, Fagerstrom KO. The Fagerstrom Test for Nicotine Dependence: a revision of the Fagerstrom Tolerance Questionnaire. Br J Addict 1991; 86: 1119-1127.

14. Gearhardt A. Role of reward pathways in appetitive drive and regulation. In: Lumeng JC, Fisher JO (eds). Pediatric Food Preferences and Eating Behaviors. Amsterdam, North Holland, Netherlands: Academic Press; 2018, pp. 111-126.

15. Miller AL, Gearhardt AN, Retzloff L, Sturza J, Kaciroti N, Lumeng JC. Early childhood stress and child age predict longitudinal increases in obesogenic eating among low-income children. Acad Pediatr 2018; 18: 685-691 https://doi.org/10.1016/j.acap.2018.01.007[published Online First: Epub Date.

16. Larsen BA, Litt MD, Huedo-Medina TB, Duffy VB. Modeling associations between chemosensation, liking for fats and sweets, dietary behaviors and body mass index in chronic smokers. Nutrients 2019; 11: 271.

17. Perkins KA, Epstein LH, Fonte C, Mitchell SL, Grobe JE. Gender, dietary restraint, and smoking's influence on hunger and the reinforcing value of food. Physiol Behav 1995; 57: 675-680. 
18. Pinheiro CR, Moura EG, Manhães AC, et al. Concurrent maternal and pup postnatal tobacco smoke exposure in Wistar rats changes food preference and dopaminergic reward system parameters in the adult male offspring. Neuroscience 2015; 301: 178-192.

19. Braun JM, Daniels JL, Poole C, et al. Prenatal environmental tobacco smoke exposure and early childhood body mass index. Paediatr Perinat Epidemiol 2010; 24: 524-534.

20. Maas CJM, Hox J. Sufficient sample sizes for multilevel modeling. Methodology 2005; 1: 86-92.
How to cite this article: Cummings J. R., Gearhardt A. N., Miller A. L., Hyde L. W., and Lumeng J. C. Maternal nicotine dependence is associated with longitudinal increases in child obesogenic eating behaviors, Pediatric Obesity. 2019;14:e12541 https://doi.org/10.1111/ijpo.12541 